\title{
Addiction Treatment and Crime Rates
}

\author{
Terry Oroszi* and Hussein Ismail \\ Department of Pharmacology and Toxicology, Boonshoft School of Medicine, Wright State University, USA
}

*Corresponding author: Terry Oroszi, Department of Master's in Pharmacology and Toxicology, School of Medicine Wright State University, USA.

\begin{abstract}
Although there was no direct connection between rehabilitation programs and crime rates, analysis and data suggested that these programs were efficient in many cases and were contributing to a decline in crime rate when implemented successfully. Addiction is defined as a physio-psychological health dysfunction characterized by compulsively craving drugs, alcohol, chemicals or any other substance that may cause harm to our bodies. It is also referred to as a "chronic state of brain disease" that may result from irrelevant use of some drugs or any other substance. Addiction and deaths related to addiction in the United States have both drastically increased during the last decade. A small portion of addicts seek medical help or rehabilitation programs, and this is the base of the problem. Whereas, the majority of addicts were more likely to be involved in crimes and offenses as a result of their addiction. The data available have found that a high prevalence of criminals was reported to be under addiction while committing the crime. This is explained by the fact that the intention of criminals to commit aggression was to get money for drugs. Further studies and researches are needed to elaborate the long-term effect of these programs and aftercare recommendations to be suggested. It's also considerable to estimate the viability of accurate statistics and addiction awareness campaigns and surveys in the United States. The focus of this review is to highlight the feasibility of rehabilitation therapies in reducing crime rates among addicts and the consequential life quality advancement.
\end{abstract}

Keywords: Addiction; Annual deaths; Crime rates; Rehabilitation therapy; Aftercare monitoring, United States

\section{Introduction}

Addiction is defined as a physio-psychological health dysfunction characterized by compulsively craving drugs, alcohol, chemicals or any other substance that may cause harm to our bodies. However, some people may have addiction to behaviors and are consequently called behavior addicted. Addiction is a chronic state of brain disease that may result from some drugs. For instance, up to 130 deaths are recorded daily in the United States due to overuse of opioids [1]. The number of drug- related annual deaths raised from around 7,000 to 68,557 deaths since $1980 \mathrm{Graph} 1$ and drugs now form the major reason behind deaths before accidents despite of the good news of a decline in drug overdose death in United States during the last month Figure 1. Addiction costs US around 740 billion dollars annually due to the huge burden of the problem [2] where data refers to 24.6 million American citizens who have used illicit drugs and 1 in 10 Americans was found to have an addiction problem during their lifetime [3]. Mortality rate related to addiction has been drastically escalating in the U.S. throughout the last years, especially those related to drug overdose in all genders where statistics have shown an increase of $24 \%$ of deaths per year between 1999 and 2017 in US according to CDC [4].
The long-term intake of drugs can cause sequential harm to the brain leading to serious mental illness as cognitive dysfunction, hallucinations, mood swings, violence, anxiety and paranoia. This cross-sectional relationship between addiction and mental illness explains the fact that addicted people are more likely to suffer from mental disorders at a rate of 2:1 compared to non-addicted people [5]. However, it's terrifying to find that among the 20 million Americans who were reported to have addiction issues, only $12 \%$ of them considered rehabilitation help [6]. The psychological consequences of addiction are explained by the changes that occur at the level of the brain due to substance abuse. This occurs after sequential uses of the drug every time the addict feels stress or pain, where they believe that the drug is the key to relieve their stress or pain. This is called "craving", where the addict encounters obsession with drug use as a salvation tool to abolish life problems and hardships along with physical and psychological sufferings.

Craving the drug or substance abused was found to increase the likelihood of the addict to engage in aggressive or risky behavior. This factor explains the elevation of crime rates in populations where addiction is prevalent Table2. It is almost impossible to 
determine the exact rate at which addiction contributes to crime records in United States. However, it is clearly considerable due to governmental records and data where drug offences accounted to up to $45 \%$ of inmates last month (August 2019) according to the Federal Bureau of Prisons (Figure 1).

Table 1: Victim's Perception of Offenders Were Under Influence of Addiction.

\begin{tabular}{|c|c|}
\hline \multicolumn{2}{|c|}{ Victim's Perception of Offenders Were Under Influence of Addiction } \\
\hline Alcohol only & $19 \%$ \\
\hline Drugs only & $11 \%$ \\
\hline Alcohol and drugs & $18 \%$ \\
\hline Neither alcohol nor drugs & $7 \%$ \\
\hline Don't know & $45 \%$ \\
\hline
\end{tabular}

Table 2: International Rate of Incarceration per 100,000.

\begin{tabular}{|c|c|}
\hline Country & $\begin{array}{c}\text { International Rate of } \\
\text { Incarceration per 100,000 }\end{array}$ \\
\hline United States & 665 \\
\hline Salvador & 418 \\
\hline Rwanda & 464 \\
\hline Russia & 383 \\
\hline Brazil & 333 \\
\hline Australia & 172 \\
\hline Spain & 127 \\
\hline China & 118 \\
\hline Canada & 114 \\
\hline France & 104 \\
\hline
\end{tabular}

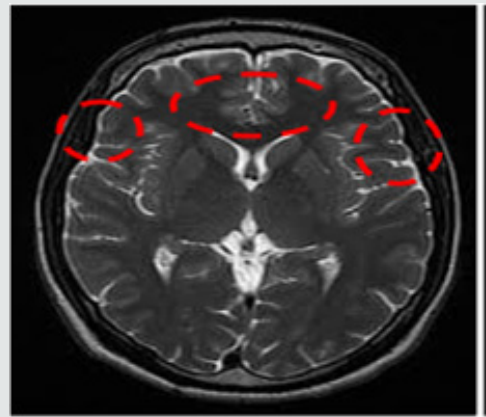

General Psychopathology

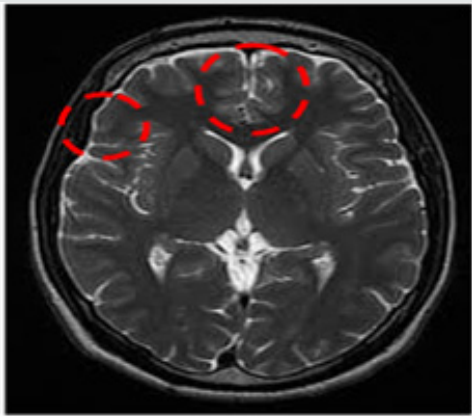

Positive Symptoms

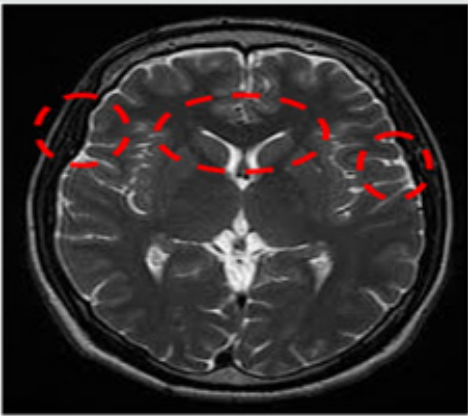

Negative Symptoms

Figure 1: General Psychopathology, Positive and Negative Symptoms Brain Activity Areas Associated with Addiction.

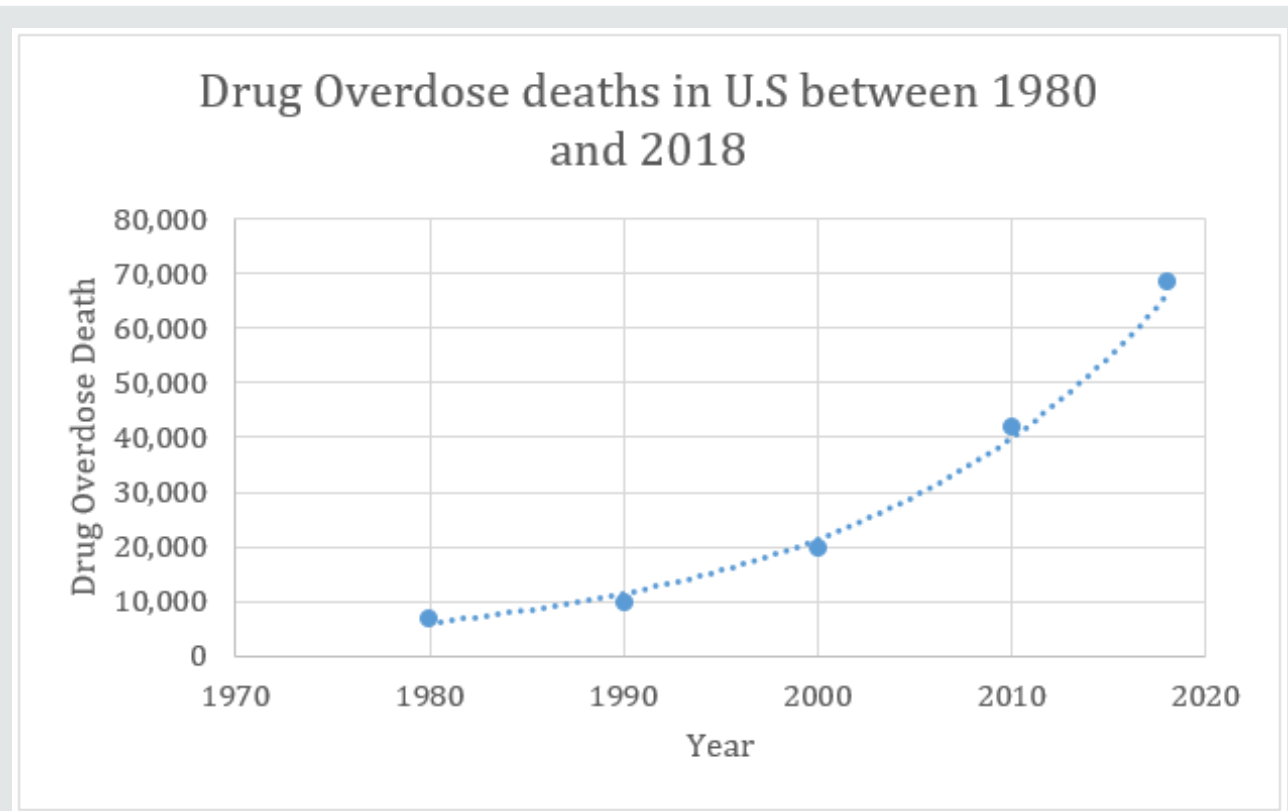

Graph 1: This graph represents drug overdose deaths in U.S between 1980 and 2018.

The detention rates in US have shown a high prevalence of drug or substance abuse among arrested offenders after drug-tests Figure 1. Interestingly, around $50 \%$ of victims reporting that they believe the criminals were under influence of addiction [7] Table 1. This prevalence is explained by the fact that the intention of criminals to commit aggression was to get money for drugs. The goal of this study is to estimate the feasibility of rehabilitation therapies in reducing crime rates among addicts and the consequential life quality advancement. It's also important to estimate the viability of accurate statistics and addiction awareness campaigns and 
surveys in the United States. Rehabilitation therapy is defined as "the analysis, diagnosis and treatment of substance use disorder through medical intervention, therapy, counseling and education." Numerous Rehabilitation therapies have been introduced and applied throughout the last decade. The therapies we are going to explore here are Cognitive Behavioral Therapy, Bibliotherapy, Gender-Specific Therapy and Contingency Management.

\section{Methods}

We identified the scope and effect of drug rehabilitation techniques on substance abuse and crime rates based on data from meta-analysis studies and Boston University investigations conducted in the context of reports and analysis relating homicides to substance abuse throughout different regions of the country. We combined these data with diverse independent data sources for drug abuse and criminal activity such as reports from Federal Bureau of Prisons (FBP) and National Institute of Drug Abuse (NIDA). We first discussed the effect of several rehabilitation techniques on addicts' psychology, social life and addiction rate scales. These scales were measured using the emotional, cognitive and behavioral alterations compared to patients' past medical and social history. Several medical techniques such as neuroimaging and clinical investigations were further used to asses these alterations. To speculate the effect of rehabilitation techniques on crime rate we used reports from U.S. governmental and security organizations. These reports were based on criminal records fluctuations and offenses being spectated at high and moderate crime areas [8]. In addition, we estimated the relevance between the cost of implementing drug rehabilitation programs and the numerous criminal annual costs on U.S. government based on data from Emory Scholars studies and New York Times investigations. These expenses were measured through-out various felonies sectors and collected records. We confined our study to United States because this country has one of the highest crimes and addiction records through-out the world and because it is one of the first countries which implemented drug rehabilitation techniques a long time ago. Various rehabilitation techniques were investigated, and we focus in our study on four of them. These techniques are Cognitive Behavioral Therapy, Bibliotherapy, Gender-Specific Therapy and Contingency Management

\section{Cognitive behavioral therapy}

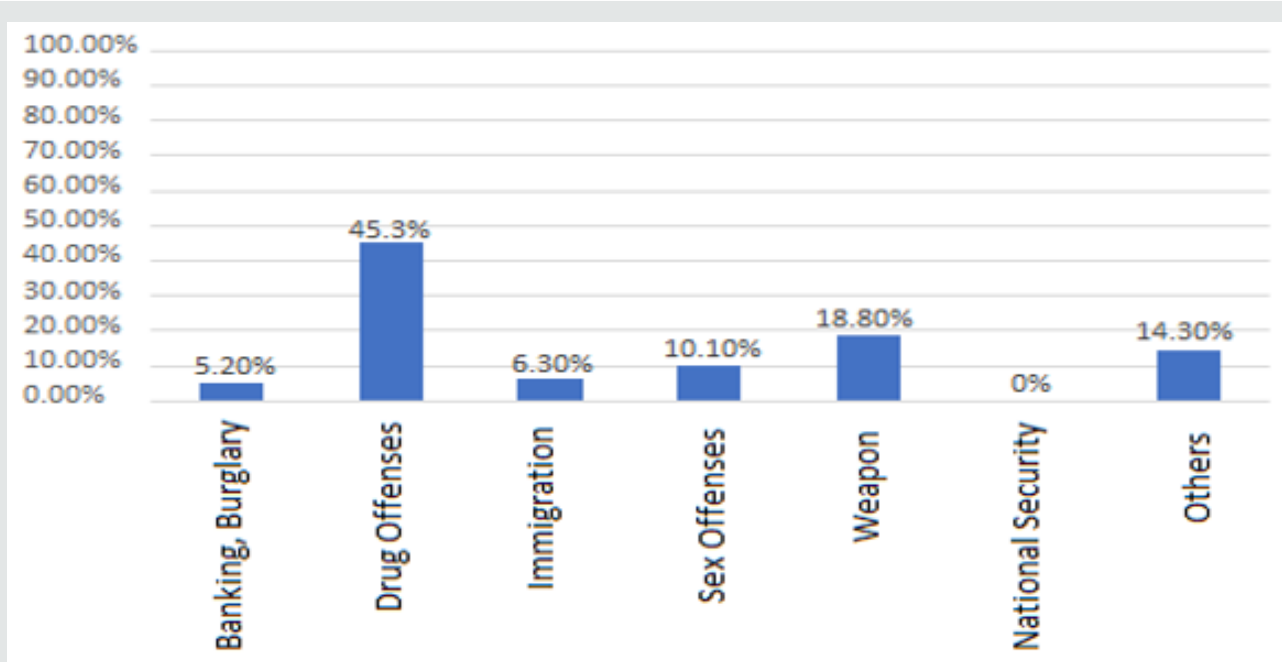

Graph 2: Statistics of inmates' percentages of different crimes in US -- Last Updated: Saturday, 31 August 2019.

Cognitive Behavioral Therapy (CBT) is a kind of psychological treatment via communication, which is built on cognitive and psychological basis of acting Graph 2. CBT aims to modify addicts' behaviors and improve their understanding of their surroundings via focusing on their mental health, emotions and satisfaction factors. CBT is then a therapy that is based on behaviorism and depends mainly on altering an individual's behavior towards being reasonable and rational. This is done by rewarding one's self to intensify a positive behavior or penalizing self to avoid or get rid of a negative behavior. Behaviors are classified into positive or negative categories according to rationality and validity [9]. However, there's also a perceptual complementary part of this therapy that focuses on psychological and intangible personality factors such as ideas, emotions and senses. It tends to identify distorted thoughts that lead to negative behavior. CBT is said to be a combination of behaviorism and perceptual therapies, where therapists not only tend to monitor and control addicts' behaviors, but also aim to reinforce their cognitive and emotional perceptions. This is due to the reality of struggling between one's expected actions and the resulted perceived actions. Addicts understand that drug abuse is a source of harm to their bodies, but they choose to engage in such behavior, although they know it would affect their lives negatively. Despite knowing this they continue the bad behavior, all the while regretting it, and having negative emotions about their own inability to stop. The conflict an addicted person can face can be seen as irrational thinking and can lead to misunderstandings or negative outcomes related to anxiety, such as depression and other several psychological problems. They believe the continued use of the drugs is the best way to overcome such difficulties and continue their abuse. This is why cognitive and perceptual part should be the backbone of the therapy. 
CBT therapy starts with a meeting between the client and the therapist in a fully comfortable situation and setting. The therapist creates a relaxing environment for the client, minimizing noise and other such distractions. To further comfort the client, and to establish trust the therapist will initiate the conversation discussing non-confrontational or threating topics. Both parties discuss the present medical history of the client and sort through negative thoughts and their understanding related to addictive behavior. The next step is directed at the client. Does the client believe they can team up with the therapist to work on altering their moods and ultimately help in getting rid of addiction? Down the road the therapist will discuss the validity of the client's thoughts and try to explain the enormous effect such dysfunctional thoughts can have on a client's life beside giving a feedback at the end of each session. Proceeding with the therapy, the addict will work through assignments at home to assess what they have discussed with the therapist and to re-evaluate their thoughts again. This can implement confidence in the client's self and boost their ability to engage in physical and psychological activities to improve their lives. CBT can have a range of 10-30 sessions based on the client's situation [10]. Each session consists of four major steps (10):

a) Identifying the problem: This step focuses on the major reasons behind the client's current situation. Both the client and therapist try to identify the situation and elaborate the consequences or possible outcomes.

b) Recognizing conceptual and emotional consequences: During this step, the therapist attempts to explore the client's thoughts, beliefs and emotions associated with their problem towards themselves and their surroundings.

c) Highlighting dysfunctional thoughts: This step helps to understand the pattern of the client's thinking that may have led to such situation. Focusing on this point can help treat the mental disorder.

d) Reformulating negative thoughts: This part of therapy aims to discuss the reality and validity of negative thoughts the addict can have. It explores the ideas rationality and attempt to in grow confidence and optimism in client's self

CBT is more than just a meeting to discuss. It's a chain of events and steps that aims to identify the client's negative thoughts and beliefs, discuss them and try to elaborate their invalidity and irrationality. This assigns the addict to study the choices at home, rethink and finally attempt to implement hopefulness and positive energy.

\section{Bibliotherapy}

Many of us have read an inspirational book about someone who had struggled against battleships of life and won the conflict. Hundreds of inspirational videos on social media and movies are inspired from books that explored the life of scrawny individuals who were overwhelmed by life difficulties and their salvation seemed to be almost impossible. These books went viral since day one of their publishing due to one major reason, which is their tremendous encouraging and motivational effect on people's lives.
This effect rises to be a type of adjuvant therapy used to treat serious issues and disorders as addiction. This is defined as Bibliotherapy.

Bibliotherapy depends on neatly selecting books by a therapist to help addicts recover from their down situations. This is a type of service that works along with other therapies to help clients identify their own problem and recognize imperfections [11]. It's a part of education and awareness that therapists use to help clients go through insight and consequently promising healing. This method also works on the psychological side of the addict to highlight problems, reasons, negative thoughts and illuminate hope that still exists despite all difficulties in life. This is extremely important to the extent that a client can now get a prescription including a book name where they can exchange prescription for the book in certain libraries in the same way that is done for medications in pharmacies. Bibliotherapy gets advantage of literature fields such as poetry, short stories and fiction that all serve to improve the client's mood in serious situations. Examples of the books which have been widely used and "prescribed" for substance abuse treatments are Jack Trimpey "Rational Recovery" The new cure for substance addiction, William White "Addiction Professionals" and Johann Hari "Chasing the Scream". The tremendous effects that bibliotherapy can have on an addict's life is interesting, ranging from fading problem importance to developing skills to solve problems and raise confidence in themselves. It's all about generating selfconfidence, education and awareness to face difficulties and stop harming self-esteem.

\section{Gender-Specific Therapy}

In the past, addiction treatment was foremost tailored for males. They were the ones most often addicted. This gender discrepancy was due to the social and moral issues that prevented women at that time to get involved with drugs, or to openly expose themselves as addicts. Moreover, the scarcity of researches conducted on females before the 20th century lead to focusing on males' behaviors, conceptions and body capacity in treatment programs and hence women were subjected to same treatment techniques. Recent researches thereafter have proven that females' bodies make them have the tendency to become physically and psychologically drug or alcohol dependent at a higher rate and lower doses than males [12]. This is because women have less total body water according to the Psychiatric Clinic of North America (PCNA) [13]. Moreover, women have fewer hepatic enzymes than men [14]. That's why their bodies metabolize drugs and alcohols at lower rates and extends the period of drugs being in their bodies. Consequently, women were found to experience adverse effects at a faster rate than men with a higher possibility of relapse. There are several reasons why men and women may need different drugaddiction treatment programs, among which are (9):

a) The respond to drugs differently: Females and males has different biological bodies; hence they metabolize drugs in different manners. According to the Journal of Substance Abuse Treatment, menstrual cycle estrogen levels affect the way the body responds to drugs and alcohol. That's why women were found to experience different levels of pleasure with illicit drugs 
than men due to their hormonal constitutes. Moreover, women are discouraged to admit being addicted or request help due to different social and psychological stigmas.

b) They have different priorities and concerns: According to the National Council of Alcoholism and Drug Dependence (NCADD), females have more tendency to endure mood problems such as anxiety, depression and psychosis. The factor which will affect their lives' quality and may interfere with their response to therapies.

c) They respond to therapy differently: Both genders encounter different hitches throughout the treatment programs. They are subjected to diverse sources of pressure and stress from their surroundings. Therefore, women and men react variously to the same therapeutic technique because they are different.

\section{Contingency Management}

Also known as the incentive's technique, Contingency Management is a kind of behavioral treatment that offers incentives for a positive behavior such as toxicology screening [15]. Otherwise, rigorous actions can be taken as a punishment for negative behavior such as missing a therapeutic appointment. These behavioral interventions have the advantage of assuring that the addict will meet treatment goals on a progressive track, which can lead to drug abstaining as soon as possible, in addition to their ability to raise the engagement rate in other therapeutic techniques as well. The unique feature about this method is that no health care professional or clinician is essentially needed to perform it. Any family member or rational relative can implement such a technique to improve the addict's quality of life and minimize further risks. Contingency Management technique has seven principles to be implemented including the following (26):

\section{Target behavior}

This principle depends on classifying the behavior being conducted by the addict. It distinguishes between the positive actions such as compliance to the instructions to be developed and the negative behavior such as the secret abuse to be halted. It aims to monitor the addict's behaviors and further detect right from wrong.

\section{Population selection}

Some addicts may find it useless or may not be interested to get engaged in therapeutic programs. That's why CM focuses on novel addicts or those with treatment program failures in the past..

\section{Booster selection}

The mainstay of CM method is the booster or reward. If this reward is inappropriate or if the addict finds it not interesting, the whole program fails. It's the heath-care team's responsibility to find out the suitable reward along with the addict in order to create curiosity in the client's self to be compliant with instruction and raise further esteem. Money has worked at most, but not in all cases.

\section{Reward extent}

This principal is crucial due to the fact of that people are diverse and have different backgrounds. The factors which determines the magnitude of the reward depends on the client's history and past commitments. Some CM programs can afford expensive rewards depending on the client's satisfaction rates.

\section{Frequency of rewards}

It's also important to determine the frequency of delivering the reward. Some CM programs offer rewards every time a positive behavior occurs. Other programs offer rewards once at a time. This all depends on the client's needs and compliance to the instructions of the programs.

\section{Time of rewards}

Another important factor is considering when to deliver the reward. Several possibilities are available, but the most effective time to give a reward is on the spot of the positive behavior. This technique creates a firm link between the behavior and the reward and establishes consistency.

\section{Therapy duration}

There's almost no exact duration for the Contingency Management Program. Ending the program occurs when the goal is achieved, which is identified as accomplishing sobriety and the desire to stop the drugs. The duration is variable depending on the clients and the quality of program.

\section{Results and Discussion}

In a 269 meta-analysis study performed in 2016 regarding Cognitive Treatment and Substance Abuse [16], CBT was found to efficiently reduce the rate of addiction to marijuana and nicotine but not alcohol and opioids. These studies along with Boston University researchers [16] found out that CBT is effective in reducing addiction rate via emotional and cognitive alteration through multiple settings rather than a single session. CBT, according to neuroimaging via MRI Figure 1, was essentially effective in decreasing the size and bounce of amygdala which is a temporal brain lobe responsible for negative thoughts as fear and anxiety as well as developing the function of prefrontal cortex responsible for rational thinking. Bibliotherapy, which is a type of psychotherapy, is a part of drug rehabilitation program which aims to boost positive energy and express feelings efficiently. In addition, it motivates addicts to seek hope in the form of stories and narratives exploring the lives of people who were the victims of their own delusions and hallucinations. According to Bibliotherapy Education Project [17], addicts who were exposed to bibliotherapy were found to be more cooperative in other adjuvant rehabilitation techniques as well as showing more enthusiasm and hope towards being cured. With the advancement of gender specific drug abuse therapy programs, the Federal law in the U.S. gave women the priority to be admitted into any publicly funded substance abuse program if they are pregnant [18]. This issue aims to save both the mother's and child's lives at once, taking into consideration the serious fact that infants could be born addicted if their moms maintain addiction during pregnancy 
or nursing period. According to Green [19], women who were involved in gender specific drug rehabilitation programs were nine times more likely to be abstinent from addiction than women who discontinued the program. On the hand, men who were involved in this program were only three times more likely to be abstinent than men who discontinued the program [20]. These findings explain the importance of gender specific programs and the tendency of women to become abstinent at a higher rate and shorter duration than men. Current research suggests that women are alike or even better than men in continuing the gender specific rehabilitation program with a higher success rate [21]. The implementation of contingency management was enough to prove that addicts can modify their behaviors when motivated in a compatible way. As a monotherapy, CM was proven to be less effective and less prevalent. It showed greater results when coupled with other therapeutic techniques [21]. However, the merit of such a method is that it's successful in cases where co-occurring diseases exist besides addiction, such as mental disorders.

Talking back about the strong association between drug's addiction and crime rates, all reports from U.S. governmental and security organizations believed that addiction is the major reason behind crime in several states. This raised up the agreement with ex-president Obama's campaign to face crime and drugs by treatment rather than jail for non-aggressive criminals [22]. This helped to declare addiction as a public health issue rather than just being a crime. Crime is not just attacking others, but it also includes self-harm or homicide. According to Swensen et. al [7], the drug rehabilitation therapies resulted in around $0.25 \%$ reduction in homicide records. Although this may not be a big contribution, it proved that rehabilitation does affect crime rate and self-harm [7]. They also found an estimate of $0.15 \%$ reduction in costs which are related to aggressive crimes (except robbery). Regarding the costs of common felonies, drug-rehabilitation techniques were associated with a reduction of $0.11 \%$ of robberies, $0.12 \%$ of motor theft and $0.09 \%$ of other related crime sectors [7]. Overall, these numbers form a reduction of $60,000 \$$ in money-driven crimes annually [7]. This is significant because such techniques will eventually reduce criminal intentions on the long-run and ultimately save money for concerned governmental institutions and affected society.

All these data explained the fact that spending a few cents on drug-rehabilitation will save dollars from associated criminal costs. According to the New York Times [23], Emory University Scholars conducted a study and proved that a $10 \%$ raise in drug rehabilitation programs rate will eventually cause a reduction of $3 \%$ in robbery rate and up to $9 \%$ in aggravated assaults rates. Billions of dollars will be saved annually from crime-related costs and social consequences if drug rehabilitation techniques are implemented and centers are built throughout the country. These techniques were not only found to reduce costs and save money, but also, they improved the quality of addicts' lives and the overall society. Consequently, crime rate would decrease, and potential criminals and their surroundings will suffer less

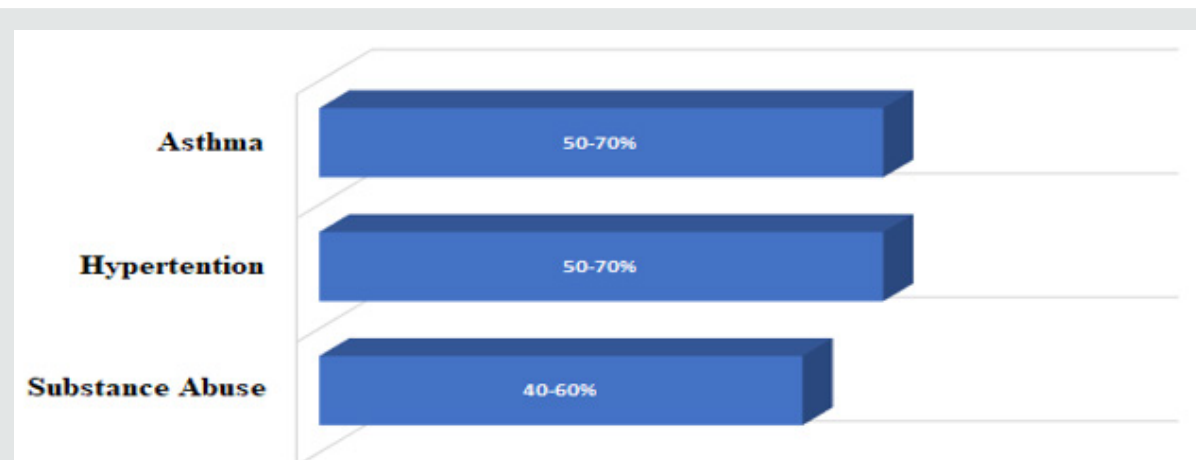

Graph 3: This graph represents comparison of relapse rates between substance use disorders and other chronic illness.

The research data from dissimilar parties [24] suggests a relapse rate of up to $30 \%$. This is enormous but still proves that $70 \%$ of rehabilitated addicts are on the right track. While other researchers suggest the relapse rate of addiction to be $40-60 \%$ at a lower rate than other chronic illnesses as asthma and hypertension (Graph 3). The provided numbers suggest that the provision and close monitoring with families and surrounding of addicts are essential to minimize the relapse rate and ensure a long-run rehabilitation effects via more effective application of rehabilitation techniques and methods. However, Niazi et.al [16], have found that the highest risk of relapse $(\sim 46 \%)$ is during the first 6 months of rehabilitation programs, whereas the risk is almost diminished after the 30th month implementation of drug rehabilitation program along with medical interventions [25]. This is serious to elaborate the importance of close monitoring during the first months of the program to assure that the client is compliant, and surroundings are supporting. It also proves that rehabilitation programs are efficient enough to prevent relapse after long-term implementation as addicts find it hard and harmful to go back to addiction as data says.

\section{Discussion}

The overall drug rehabilitation techniques mentioned above which focus on psychological intervention are synergistic to drug interventions, such as methadone and buprenorphine, and facilitates the mission of treatment faster. Focusing on mental wellness and the psychological side of addicts is equal to or even more important than medical interventions alone. This is due to the 
human nature of addicts that is controlled by emotions, instincts and psychological drives. All researches conducted agreed on the definition of addiction as a "brain disease" which creates craving towards a specific drug, chemical or behavior. This is important to drive the drug rehabilitation techniques towards the mental and psychological directions of an addict. These techniques were efficient in helping the addict to cope with tension, avoiding upsetting emotions and raising emotional intelligence. They urge

Table 3: Variables Associated with Relapse to Addiction. the addict to be smart and alert enough to take a responsible action whenever bad situations are encountered instead of giving up on life and losing hope. The major determinant for relapse is the state of withdrawal symptoms that could be painful and even fatal if not controlled well [20]. However, there are also other several factors and determinants for relapse such as employment status, friends abusing drugs, family support, social and economic status (Table 3).

\begin{tabular}{|c|c|}
\hline \multicolumn{2}{|c|}{ Variables Associated with Relapse to Addiction (P) } \\
\hline Individual Factors & 0.48 \\
\hline Family Factors & 0.23 \\
\hline Occupational Factors & 0.2 \\
\hline Surrounding Factors & 0.09 \\
\hline
\end{tabular}

${ }^{a}$ Abbreviations Probability (P)

This explains the importance of closely monitoring and supervising of client's status and surroundings throughout the rehabilitation program. Moreover, all the new or suggested rehabilitation techniques show the importance of psychological and mental wellness of the addict as they focus mainly on the zone of cognitive health [26]. These methods include yoga, meditation, spiritual and religious methods (i.e. Catholic drug rehabilitation), balanced diets, message therapy and art therapy. These newly introduced and suggested holistic techniques focus on the relaxation and wellbeing of the addict's body and brain. This is essential for the addict to relieve stress and avoid troublesome which may eventually stimulate the negative personality when hope in life and happiness is gradually fading. This drives each person to discover a new passion, create healthy relationships and/or friendships to find out that drugs are not the last ditch to survive and that doing what we love is the key towards salvage.

\section{Conclusion}

Based on the given data and available statistics, rehabilitation programs were a major salvage for millions of addicts inside and outside the United States. This redemption exit was either to evade from prosecution or to improve their own quality of life and engage in social activities. These programs were also associated with positive effects on various types of crimes and crime rates in the nation (decline in crime rate). Drug Rehabilitation Programs were effective in reducing crime-related costs by around 9 million dollars annually whereas the annual cost of implementing drug rehabilitation programs is approximately 1 million dollars (15) [12].

This proves that the advantage of rehabilitation programs outweighs the annual crime costs. All available data and researches didn't find yet a direct explicit bond between drug rehabilitation programs and crime rates fluctuations. All the results retrieved, and conclusions made were based on analysis and expectations. Extensive research in such field should be conducted and further relevant data should be collected to elaborate the feasibility of drug rehabilitation programs. However, the scarcity of research does not deny the effectiveness of these programs and their efficiency in improving the clients' quality of life and behaviors. The governmental security agencies have also reported reduction in crime rates where drug rehabilitation centers were found $[27,28]$.

Therefore creating a recovery sauna will ultimately reduce crime rate and enhance the overall security of the society. These centers should be expanded more intensively all around the nation with each state taking into consideration the heterogeneity of population and diversity of backgrounds and inducements with considering several smart advertising campaigns to reach addicts and attract them to join. Moreover, these centers should establish a long-term post recovery monitoring system to track the sobriety of their clients and reduce relapse rate besides offering safeguarding duties all over the facilities and client's lodgings. Aftercare programs are essential to ensure the success of rehabilitation and maintain sobriety among the clients. The rehabilitation center's staff, and the monitoring health care professionals shall provide the client with a list of recommended instructions to pursue a better lifestyle. Therefore, the scope of future researches should focus on expanding the area of drug rehabilitation centers and developing after care programs and monitoring techniques.

\section{References}

1. (2015) Bibliotherapy: Definition and history.

2. (2019) Federal Bureau of Prisons.

3. (2019) Substance Abuse and Mental Health Issues.

4. Gender Specific Treatment Programs in Texas. Nova recovery center, USA.

5. (2017) Data Briefs. pp. 294.

6. Allkindsoftherapy \& All Kinds of Therapy Nova recovery Bibliotherapy Resource - Addiction/Sobriety.

7. Bondurant R S, Lindo M J, Swensen DI (2016) Substance Abuse Treatment Centers and Local Crime.

8. Bureau of Justice Statistics. 
9. Clancy CCC, Digital Marketing (2019) Gender-Specific Treatment for Substance Abuse Journey Pure.

10. (2019) Cognitive behavioral therapy.

11. Contingency Management (CM).

12. Reuters (2012) Drug Treatment Could Help Lower U.S. Crime Rates, Data Suggests.

13. Frakt A (2017) Spend a Dollar on Drug Treatment and Save More on Crime Reduction.

14. Hardey Reviewed S, Thomas S (2019) The Effectiveness of Holistic Treatment Techniques in Drug Rehab.

15. Hofmann SG, Asnaani A, Vonk IJJ, Sawyer AT, Fang A (2012) The Efficacy of Cognitive Behavioral Therapy: A Review of Meta-analyses. Cognit Ther Res 36(5): 427-440.

16. Kassani A, Niazi M, Hassanzadeh J, Menati R (2015) Survival Analysis of Drug Abuse Relapse in Addiction Treatment Centers. Int J High Risk Behav Addict 4(3): e23402.

17. Lopez (2017) How Obama quietly reshaped America's war on drugs.

18. Mason Liam, Peters RE, Dima Danai,Williams (2015) Cognitive Behavioral Therapy Normalizes Functional Connectivity for Social Threat in Psychosis. Schizophr Bull 42(3): 684-692.
19. Melemis S (2019) Relapse Prevention Plan and Early Warning Signs.

20. National Institute on Drug Abuse (2017) Trends \& Statistics.

21. National Institute on Drug Abuse (2015) Nationwide Trends.

22. National Institute on Drug Abuse (2007) Reading To Recover: Exploring Bibliotherapy as a Motivational Tool for Recovering Addicts.

23. National Institute on Drug Abuse (2018) Sex and Gender Differences in Substance Use Disorder Treatment.

24. Carla A Green (1997) Gender and Use of Substance Abuse Treatment Services. NIAAA Publications.

25. Patterson E, Ncc (2017) Contingency Management (CM) Therapy for Addiction Treatment.

26. Rudd RA, Aleshire N, Zibbell JE, Gladden RM (2016) Increases in Drug and Opioid Overdose Deaths - United States, 2000-2014. MMWR 64(50): 1378-1382.

27. Shafiei E, Hoseini A F, Bibak A, Azmal M (2014) High risk situations predicting relapse in self-referred addicts to bushehr province substance abuse treatment centers. Int J High Risk Behav Addict 3(2): e16381.

28. TB (2019) Why Alcohol and Drugs Are Factor in a Large Percentage of Crimes.

\section{(a) (1) \\ This work is licensed under Creative Commons Attribution 4.0 License}

To Submit Your Article Click Here:

Submit Article

DOI: $10.32474 /$ RRHOAJ.2019.04.000186

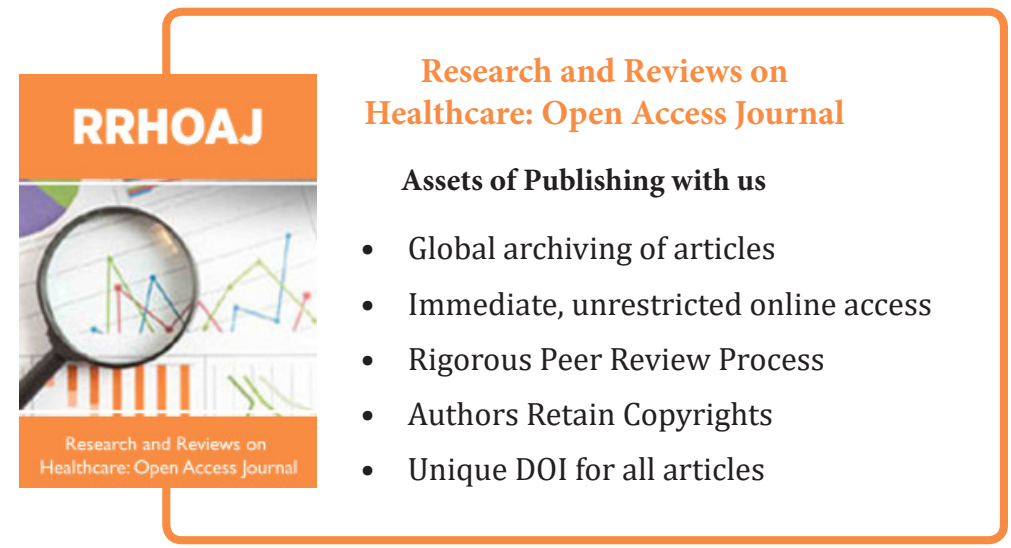

